\title{
Influence of Shape and Piezoelectric-Patch Length on Energy Conversion of Bluff Body-Based Wind Energy Harvester
}

\author{
Changjiang Zhang, ${ }^{1,2}$ Lin Ding $\mathbb{D}^{1,2}$ Lin Yang, ${ }^{1,2}$ Zuomei Yang, ${ }^{1,2}$ Zesheng Yang, ${ }^{1,2}$ \\ and Li Zhang ${ }^{1,2}$ \\ ${ }^{1}$ Key Laboratory of Low-grade Energy Utilization Technologies and Systems of Ministry of Education, Chongqing University, \\ Chongqing 400044, China \\ ${ }^{2}$ School of Energy and Power Engineering, Chongqing University, Chongqing 400044, China
}

Correspondence should be addressed to Lin Ding; linding@cqu.edu.cn

Received 29 January 2020; Revised 18 May 2020; Accepted 16 June 2020; Published 14 July 2020

Academic Editor: Lingzhong Guo

Copyright (c) 2020 Changjiang Zhang et al. This is an open access article distributed under the Creative Commons Attribution License, which permits unrestricted use, distribution, and reproduction in any medium, provided the original work is properly cited.

\begin{abstract}
The technology of scavenging ambient energy to realize self-powered of wireless sensor has an important value in practice. In order to investigate the effects of piezoelectric-patch length and the shape of front bluff body on energy conversion of the wind energy harvester by flow-induced vibration, the characteristics of a piezoelectric wind energy harvester based on bluff body are experimentally studied in this work. Four different section shapes of the bluff body, including triangular cylinder, trapezoidal cylinder, reverse trapezoidal cylinder, and square cylinder, are tested. The piezoelectric patch is attached on the leeward side of the bluff body. The lengths of piezoelectric patch are considered as 1.0D-1.4D ( $D$ is the characteristic length of the bluff body). It is found that the length of the piezoelectric patch and the shape of the front bluff body play a vital role in improving the performance of wind energy harvester. For the reverse trapezoidal cylinder and square cylinder, the back-to-back vortex-induced vibration (VIV) and galloping phenomenon can be observed. In addition, the energy harvesting performance of the reverse trapezoidal cylinder piezoelectric harvester is the best. The maximum average peak voltage of $1.806 \mathrm{~V}$ and the output power of $P=16.3 \mu \mathrm{W}$ can be obtained when external resistance and the length of piezoelectric patch are $100 \mathrm{~K} \Omega$ and $1.1 D$, respectively.
\end{abstract}

\section{Introduction}

In recent years, microelectromechanical systems (MEMS) have made great progress and sensors are gradually becoming intelligent, miniaturized, and wireless-networked [1-4]. Energy supply is increasingly becoming a bottleneck in its development. Traditional batteries and power wires cannot satisfy the functional requirements because of the short lifespan, complex route, and other shortcomings. Meanwhile, the development of large-scale and low-power integrated circuit technology makes the power consumption of electronic products continue to decrease, which has been dropped to milliwatt or even microwatt level [5]. Therefore, the technology of scavenging ambient energy to realize selfpower of electronic equipment has been proposed and widely concerned. Traditional hydroelectric generators and wind turbines, due to the mechanical losses of bearings, have caused lower efficiency during miniaturization [6]. However, the energy harvester based on flow-induced vibration (FIV) is simple in structure and easy to miniaturize and has no rotating parts, which has attracted a large amount of research [7-12].

FIV is a very complex physical phenomenon. When fluid flows around the structure, vortices are generated, and the structure is subjected to periodic forces caused by the vortices. The elastic structure may vibrate under the action of this force; this fluid-structure interaction is called FIV [13-18]. In particular, when the frequency of the fluid force approaches the natural frequency of the structure, the structure will generate a large vibration. Based on this phenomenon, many flow-induced vibration energy harvesters have been developed. A typical energy harvester is 
Vortex-Induced Vibration for Aquatic Clean Energy (VIVACE) converter, which utilizes FIV to convert mechanical energy into electricity energy through the principle of electromagnetic induction [19-21].

In a subsonic flow, bluff body may induce vortices shedding. Due to the alternating shedding of the vortex, the disturbance of the structure wake is more intense [22]. Thus, part of energy harvester is designed to place flexible piezoelectric materials in the wake of the cylinder. Taylor et al. [23] firstly proposed an energy harvesting device that uses the PVDF (polyvinylidene fluoride) flexible piezoelectric material to harvest energy from ocean and river. It consisted of a piezoelectric membrane or "eel," which is fixed in the wake of bluff body. In the same year, Allen and Smith [24] found that resonance occurs when the vibration frequency of the "eel" closes to the vortex shedding frequency. Resonance is considered to be the optimal coupling for energy harvesting, and the damping effect of the "eel" on the flow field is negligible. Cellini et al. [25] proposed a water energy harvest device; they selected ion-exchange polymer metal composite on the piezoelectric material. Their experimental results showed that the efficiency of the output power is related to the load of the external circuit and the incoming flow velocity. The power of the device is $0.1 \mu \mathrm{W}$ at a current velocity of $1.1 \mathrm{~m} / \mathrm{s}$ when the parameters are optimal. PZT ( $\mathrm{Pb}$-based lanthanum-doped zirconate titanate) piezoelectric materials are also widely used in energy harvesting. Song et al. [26] presented an upright vortex-induced piezoelectric energy harvester (VIPEH), which is consisted of a piezoelectric (PZT) beam with a circular cylinder. At a resonance speed of $0.35 \mathrm{~m} / \mathrm{s}$, the maximum power is $84.49 \mu \mathrm{W}$ and the energy density is $60.35 \mathrm{~mW} / \mathrm{m}^{2}$. Hu et al. [27] developed a theoretical model to evaluate the optimal location of VIPEH in bluff body wake, which was verified by a series of experiments.

In addition, many wind-induced vibration energy harvesters have been proposed [12]. Lots of studies proved that it is feasible to harvest wind-induced vibration energy through piezoelectric materials [28-34]. However, improving the electromechanical conversion efficiency and power density for wind-induced vibration energy harvesters is an urgent problem to be solved. $\mathrm{Hu}$ et al. [35] experimentally evaluated the ability of attachments with different cross-section shapes on the circular cylinder to improve the efficiency of VIPEH. It was found that the installation of the triangular attachment on the cylindrical surface has the highest energy harvesting efficiency. Zhang et al. [36] conducted optimal experiments of VIPEH with different interference cylinder sections shapes. The results showed that, compared with the noninterfering cylinder, the energy harvesting efficiency can be significantly improved when the cross-sectional shape of the interference cylinder is square. When the wind speed is 2.36 and the spacing ratio is 0.9 , the average power can reach $803.4 \mathrm{~mW}$, and the synchronization area is increased by $380 \%$. Dai et al. [37] investigated the influence of the bluff body installation on energy harvesting for designing efficient energy harvesters. Wang et al. [38] proposed a method to improve the efficiency of VIPEH by using Y-shaped attachment on bluff body. Therefore, improving energy harvester efficiency is of great significance for the application of VIPEH to practical engineering. More information on energy harvesting by flow-induced vibration can been found in the review article by Wang et al. [12].

In this study, the performance of harvester with different lengths of piezoelectric patch attached on the bluff body is experimentally investigated in an open-circuit wind tunnel. The influence of the cross-sectional shape of bluff body on the energy conversion is studied as well. Experimental wind speed ranges from $2 \mathrm{~m} / \mathrm{s}$ to $13 \mathrm{~m} / \mathrm{s}(R e=6800 \sim 44000)$. The energy output of the piezoelectric patch in FIV is analyzed. The piezoelectric material of PVDF is applied in the experimental tests. The paper is structured as follows: First, Section 2 introduces the theory and model of piezoelectric energy harvesting. Section 3 describes the experimental equipment and some specific parameters. Then, Section 4 discusses the main experimental results of this study. Finally, Section 5 summarizes the most important results and conclusions.

\section{Theory and Model of Piezoelectric Energy Harvesting}

In this study, the piezoelectric patch with two ends, one fixed and the other free, is considered. Periodic forces on the piezoelectric patch are caused by vortices shedding, resulting in the deformation of the piezoelectric patch. As shown in Figure $1, l$ and $W(x)$ are the length and deflection of the piezoelectric patch, respectively. The bluff body is fixed by connecting the center (point $O$ ) of the bluff body to the support structure in the wind tunnel. Since the thickness of the piezoelectric patch is negligible compared with the width of the piezoelectric patch, it can be treated as a thin plate. Considering that it vibrates only in the $z$ direction, there is only a displacement in the $x$ direction. Therefore, the strain $\left(\varepsilon_{x}\right)$ and stress $\left(\sigma_{x}\right)$ in the $x$ direction can be expressed as

$$
\begin{aligned}
\varepsilon_{x} & =z \frac{\partial^{2} W}{\partial x^{2}}=z k_{x}, \\
\sigma_{x} & =\frac{-Y z^{\prime}}{1-v^{2}} k_{x},
\end{aligned}
$$

where $z^{\prime}$ is the coordinate value of the piezoelectric patch along the thickness direction; $W$ is the deflection in the $z$ direction, the second derivative of which is the curvature $k_{x}$ of the $x$ direction; $Y$ represents Young's modulus of the piezoelectric patch; and $v$ is the Poisson ratio. For the thin plate, the strain should be linearly distributed along the normal direction, and the strain on the upper and lower surfaces of the plate is the largest; that is, $z^{\prime}=h / 2$.

The deflection of the piezoelectric patch is

$$
W=W(x) \sin (\omega t)
$$

The constitutive equation for energy collection of onedimensional transverse strain (tensile direction of piezoelectric patch) is as follows: 


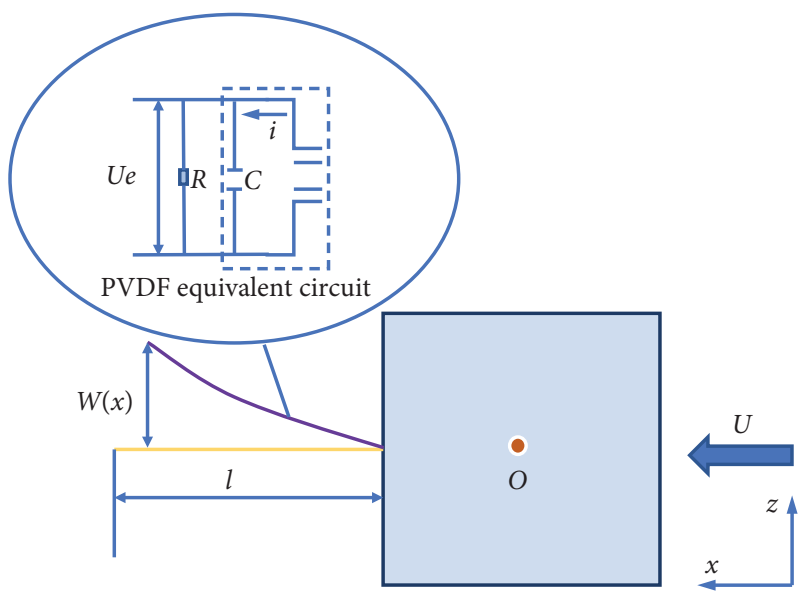

Figure 1: Piezoelectric patch vibration model.

$$
\begin{gathered}
\varepsilon_{x}=s_{11} \sigma_{x}+d_{31} E_{z}, \\
D_{z}=d_{31} \sigma_{x}+E_{z} \varepsilon_{33},
\end{gathered}
$$

where $E_{\mathrm{z}}$ is the electric field along the thickness direction of the piezoelectric patch; $\varepsilon_{33}$ is the dielectric constant under a certain stress; $D_{z}$ is the charge density per unit area. Equation (3) can be rewritten as

$$
\sigma_{x}=\frac{\left(\varepsilon_{x}-d_{31} E_{z}\right)}{s_{11}} \text {. }
$$

Plugging equation (5) into equation (4), the charge density produced by the piezoelectric patch is

$$
D_{z}=\frac{d_{31}}{s_{11}} \varepsilon_{x}+\left(\varepsilon_{33}-\frac{d_{31}^{2}}{s_{11}}\right) E_{z} .
$$

By using the surface integral of electric displacement, the expression of the electric charge produced by the piezoelectric patch is obtained as follows:

$$
Q=\int_{A} D_{A} S d S=b \int_{l_{0}}^{l_{1}}\left(d_{31} \sigma_{x}+E_{z} \varepsilon_{33}\right) d x
$$

where $l_{0}$ and $l_{1}$ are the positions of the root and the end of the piezoelectric plate and $S$ is the area of the piezoelectric electrode, which can be obtained from equation (3):

$$
E_{z}=\frac{\varepsilon_{x}-s_{11} \sigma_{x}}{d_{31}}
$$

Bringing equation (8) into equation (7) gives

$$
Q=\left[\frac{b E h d_{31}}{2}\left(s_{11}-\frac{1}{1-v^{2}}\right)+\frac{h b \varepsilon_{33}}{2 d_{31}}\right] \times\left[\varphi\left(l_{1}\right)-\varphi\left(l_{0}\right)\right] \text {, }
$$

where $\varphi(l, t)=\partial W(x, t) / \partial x$ is the flexibility of the thin plate and $\varphi\left(l_{0}\right)$ and $\varphi\left(l_{1}\right)$ are the corresponding values of the root and the end of the plate at the time $t$, which can be obtained by experiments.

The equivalent circuit of piezoelectric harvester is shown in Figure 1 as well, where $R$ and $U_{\mathrm{e}}$ represent external load resistance and external voltage, respectively, in which the dotted wire frame is the circuit model of piezoelectric patch.
From Figure 1,

$$
i=\frac{C d U_{e}}{d t}+\frac{U_{e}}{R} \text {. }
$$

Meanwhile,

$$
i=\frac{d Q}{d t}=Q \omega \cos (\omega t)
$$

Bringing equation (11) into equation (10), we get

$$
C \frac{d U_{e}}{d t}+\frac{U_{e}}{R}=Q \omega \cos (\omega t) \text {. }
$$

The solution of the equation is

$$
U_{e}=A e^{-(t / R C)}+e^{-(t / R C)} \frac{Q \omega}{C} \int e^{t / R C} \cos (\omega t) d t .
$$

With the increase of time, the general solution $A e^{-(t / R C)}$ tends to 0 . The voltage $U_{\mathrm{e}}$, therefore, is mainly determined by the special solution; that is,

$$
U_{e}=\frac{Q}{C} \sqrt{\frac{R^{2} C^{2} \omega^{2}}{1+R^{2} C^{2} \omega^{2}}} \sin (\omega t+\alpha) .
$$

Output power is

$$
P=\frac{U_{e}^{2}}{2 R}=\frac{R^{2} \omega^{2}}{2 R\left(1+R^{2} C^{2} \omega^{2}\right)} .
$$

The power in a fluid can be calculated as follows:

$$
P_{\text {fluid }}=\frac{1}{2} \rho U^{3} D L \text {. }
$$

The conversion efficiency is defined as

$$
\eta=\frac{P}{P_{\text {fluid }} \times \text { Betz_Limit }},
$$

where Betz Limit is $16 / 27$.

\section{Experimental Setup}

As can be seen from Figure 2, the experiment is carried out in the wind tunnel with a $30 \mathrm{~cm} \times 40 \mathrm{~cm}$ test section. The models of the FIV cylinder are presented in Figure 3. There are four different section shapes of the bluff body in our work: triangular cylinder, trapezoidal cylinder, reverse trapezoidal cylinder, and square cylinder. The length of the cylinder is $L=215 \mathrm{~mm}$, characteristic length is $D=50 \mathrm{~mm}$, and $d=1 / 2 D$ (short side of the trapezoidal cylinder). The bluff body locates upstream; the piezoelectric patch is mounted on the leeward side of the bluff body, which is fixed with splint and connected with a resistance. It should be noted that the optimal resistance value will change when the lengths of the piezoelectric patch are different. For better output measurement, resistance value of $R=100 \mathrm{~K} \Omega$ is considered. In order to realize the conversion of mechanical energy into electrical energy, a flexible piezoelectric material such as PVDF was selected. PVDF is easily deformed under the action of wind force. The detailed parameters of PVDF are listed in Table 1. The working length of the PVDF piezoelectric patch ranges from $1 D$ to $1.4 D$, the width is 
$45 \mathrm{~mm}$, and the thickness is $200 \mu \mathrm{m}$. The voltage of the resistance, the vibration of the piezoelectric patch, and wind speed are measured by Agilent U2300A, laser displacement sensor, and hot-wire anemometer, respectively.

\section{Results and Discussion}

4.1. Influences of Wind Speed on Energy Harvesting. A square cylinder with a PVDF piezoelectric patch of size $l=1.1 D$ is analyzed firstly as a reference case. Figure 4 presents the output voltage of the piezoelectric patch under different wind speeds. The peak value of voltage increases gradually as the wind speed increases from $3 \mathrm{~m} / \mathrm{s}$ to $12 \mathrm{~m} / \mathrm{s}$. At wind speed $U=6 \mathrm{~m} / \mathrm{s}$, the maximum voltage reaches $1.4 \mathrm{~V}$, which is about one order larger than that of $U=3 \mathrm{~m} / \mathrm{s}$. The maximum voltage reaches $2.5 \mathrm{~V}$ at $U=12 \mathrm{~m} / \mathrm{s}$, which is twice as much as $U=6 \mathrm{~m} / \mathrm{s}$. This can be attributable to the enhancement of wake disturbance, and the larger the wind speed is, the stronger the wake disturbance is. The deformation of piezoelectric patch increases with the increase of wake intensity. These results illustrate that wind speed will significantly affect the voltage output of the harvester.

For the purpose of further reflecting the voltage output characteristics of the harvester, the average value of the peak voltage $\left(V_{\text {peak }}\right)$ is obtained by calculating the mean of the absolute values of all peak voltages in this study. The power is calculated through $P=U^{2} / 2 R$. Figure 5 shows the mean peak voltage and power of harvester. The voltage growth process is relatively slow as the wind speed increases from $2 \mathrm{~m} / \mathrm{s}$ to $3.6 \mathrm{~m} / \mathrm{s}$; the output voltage is low in this wind speed range. At $U=4 \mathrm{~m} / \mathrm{s} \sim 6.5 \mathrm{~m} / \mathrm{s}$, the voltage increases rapidly. This is caused by the lock-on between vortices shedding and the vibration of cylinder. The piezoelectric patch, at the same time, is driven to produce a large deformation. The maximum output voltage and power of the square cylinder-piezoelectric patch system of $l=1.1 D$ at a wind speed of $6.3 \mathrm{~m} / \mathrm{s}$ are $1.065 \mathrm{~V}$ and $0.57 \mu \mathrm{W}$, respectively. Then the voltage drops slightly. The square cylinder is in a transition phase. However, the mean peak voltage curve shows a rapid growth process when the wind speed is beyond a critical value of $U_{c i}=8.53 \mathrm{~m} / \mathrm{s}$. The piezoelectric patch near the fixed end begins to vibrate, so that the vibration amplitude of the piezoelectric patch is greatly increased. This is because the occurrence of vibration causes a large vibration of the piezoelectric patch. A VIV and galloping phenomenon are observed. At $U=12.90 \mathrm{~m} / \mathrm{s}$, the maximum voltage and output power are $1.595 \mathrm{~V}$ and $12.70 \mu \mathrm{W}$, respectively.

Figure 6 shows the voltage frequency spectra for the square cylinder-piezoelectric patch system of $l=1.1 D$. It is noted from Figure 6 that the output voltage of the base frequency is $20 \mathrm{~Hz}$ at $U=3 \mathrm{~m} / \mathrm{s}$. There is a small peak at $10 \mathrm{~Hz}$. This is due to the fact that the piezoelectric patch has only the tail flutter at this time, which causes lots of nonlinear fast vibrations. At $U=6 \mathrm{~m} / \mathrm{s}$, the dominant frequency of the output voltage is $18 \mathrm{~Hz}$. The frequency spectrum shows a small peak at $36 \mathrm{~Hz}$, which means that the vibration is unstable. The small second peak is the previous transition phase, which is featured by a high-frequency vibration and small amplitude. At $U=9 \mathrm{~m} / \mathrm{s}$, the dominant frequency of the output voltage is maintained at $18 \mathrm{~Hz}$. There are many similar peaks near the base frequency. This is due to the fact that the vibration of the piezoelectric patch is not as regular as the case of $U=6 \mathrm{~m} / \mathrm{s}$. At $U=12 \mathrm{~m} / \mathrm{s}$, the base frequency is $15 \mathrm{~Hz}$, and the amplitude of the base frequency is significantly reduced. It represents that the amplitude response of harvester evolved into galloping, featured by a low-frequency vibration and large amplitude.

4.2. Effects of Length of Piezoelectric Patch on Energy Output. In order to optimize the energy harvesting of the square cylinder-piezoelectric patch system, the performances of piezoelectric patch with different lengths are presented in Figures 7 and 8 . For the cases of $l=1.3 D$ and $1.4 D$, the voltage increases slowly with the wind speed, and its voltage output is higher than the case with $l=1.0 D, l=1.1 D$, and $l=1.2 \mathrm{D}$ at low wind speed. At medium and high wind speeds, the output voltages for the cases of $l=1.3 D$ and $1.4 D$ are significantly smaller than those of $l=1.0 D, l=1.1 D$, and $l=1.2 D$. The VIV and galloping phenomenon are observed at $l=1.0 D, l=1.1 D$, and $l=1.2 D$, and the voltage increases monotonically with wind speed when the wind velocity beyonds critical wind speed $\left(U_{c i}\right)$. Critical wind speed $\left(U_{c i}\right)$ is the minimum velocity required for galloping. For $l=1.0 D$, $1.1 D$, and $1.2 D$, the resonant wind speed decreases as the length of the piezoelectric patch increases. After the resonance, there will be a relatively stable transition phase of the output voltages and power. When the wind speed continues to increase until beyond critical wind speed $\left(U_{c i}\right)$, the whole piezoelectric patch starts vibration, and the output voltages and power become larger. The piezoelectric patch of $l=1.1 D$ produces the best performance for harvesting power when the wind speed exceeds $6.3 \mathrm{~m} / \mathrm{s}$. The maximum voltage and power of the square cylinder-piezoelectric patch system can reach $1.596 \mathrm{~V}$ and $12.70 \mu \mathrm{W}$, respectively. It should be mentioned that the VIV and galloping phenomenon result in an improved efficiency of the harvester and the bandwidth of harvester depending on the length of piezoelectric patch.

\subsection{Effects of Bluff Body Cross-Sectional Shape on Voltage} Output. Figures 9 and 10 show the effect of geometry on the energy output of the harvester. The length of piezoelectric patch is $1.1 D$, which is the best value from the results of section 4.2. It can be seen from Figures 9 and 10 that the average peak voltage and power trend of triangular cylinder are similar to those of the trapezoidal cylinder, which approaches linear growth.

The length of the leeward edge of triangular and trapezoidal cylinder is longer than the windward side. The flow boundary layers separate at the edge of the leeward side, and the separation point is no longer affected by the bluff body. Therefore, the shedding vortex directly acts on the piezoelectric patch; the voltage and power output of the two cylinders are similar. The difference is that the windward side of the trapezoidal cylinder will stimulate the separation of the boundary layer, and energy output of the trapezoidal cylinder-piezoelectric patch system is higher at low wind speeds. 

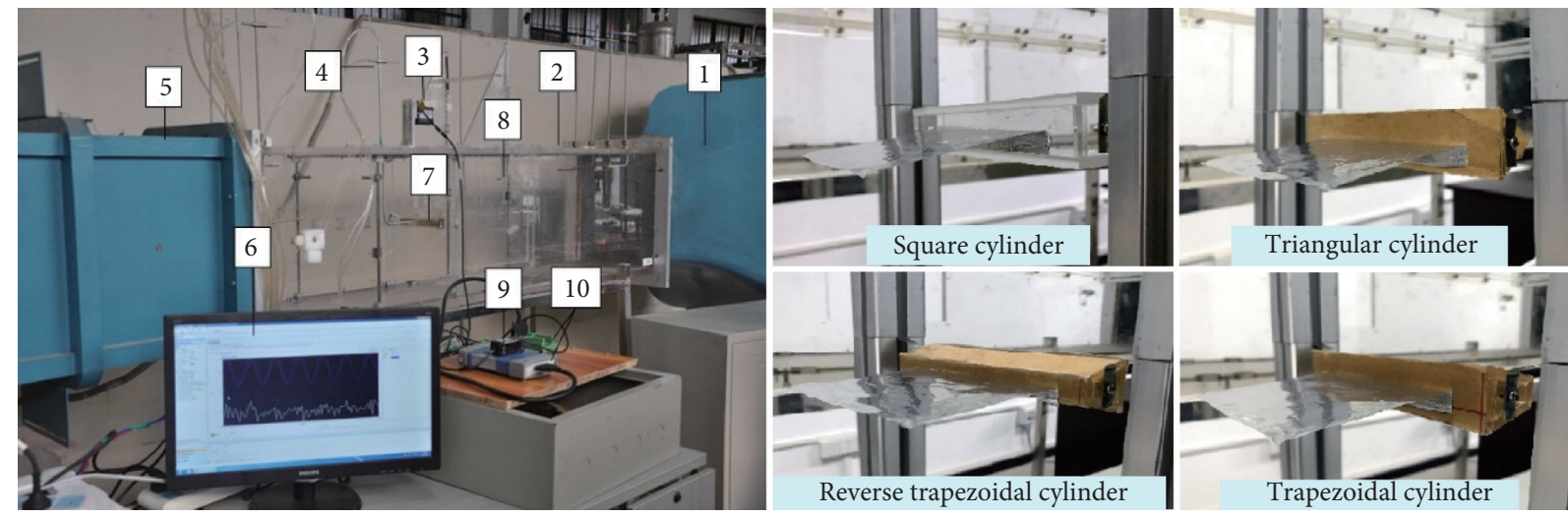

Figure 2: Wind tunnel. 1, wind tunnel test section; 2, fixed device; 3, square cylinder-piezoelectric patch system; 4, laser displacement sensor; 5, data acquisition instrument; 6, computer; 7, entrance of wind tunnel; 8, air-induced section.

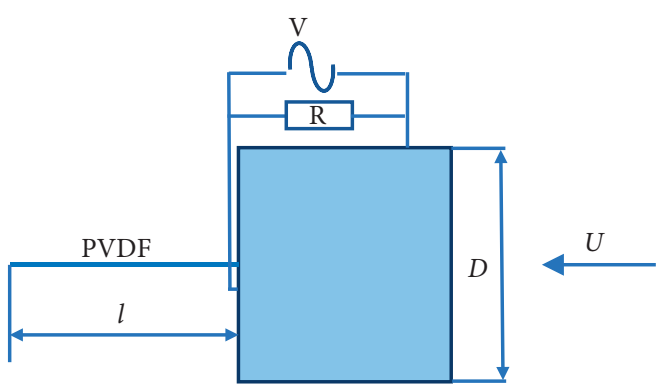

(a)

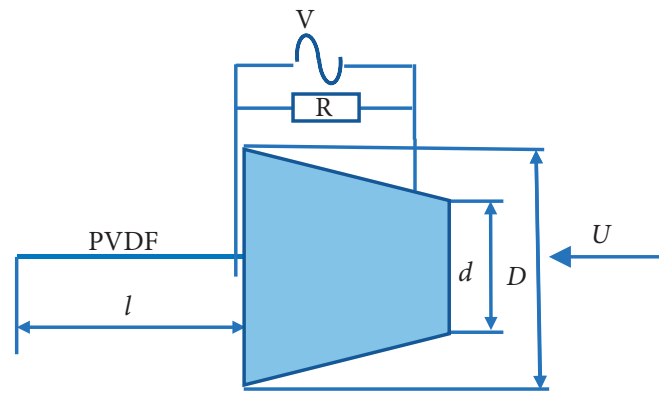

(c)

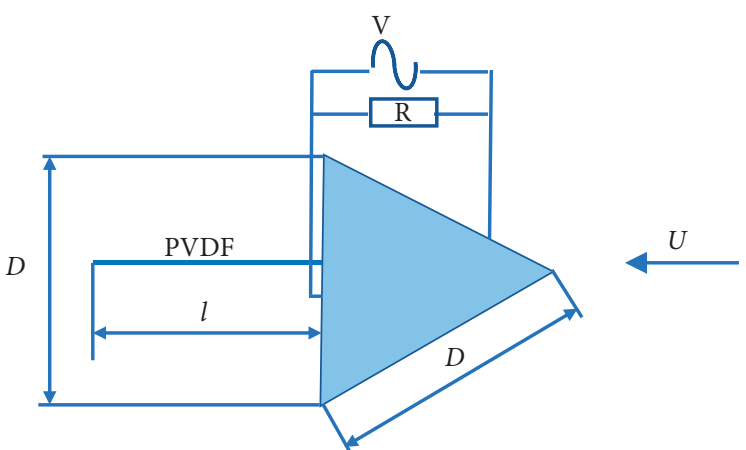

(b)

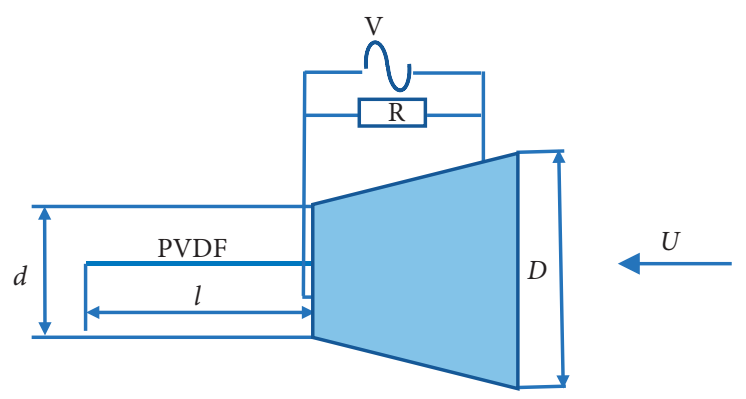

(d)

FIGURE 3: Physical model of bluff body-piezoelectric patch system: (a) square cylinder; (b) triangular cylinder; (c) trapezoidal cylinder; (d) reverse trapezoidal cylinder.

TABLE 1: Parameters of PVDF piezoelectric patch.

\begin{tabular}{lc}
\hline Parameter & Value \\
\hline Thickness tolerance & $\pm 3 \% \sim \pm 5 \%$ \\
Piezoelectric strain constant $d_{31}$ & $17 \pm 2.1 \mathrm{PC} / \mathrm{N}$ \\
Piezoelectric strain constant $d_{33}$ & $21 \mathrm{PC} / \mathrm{N}$ \\
Relative dielectric constant & $9.5 \pm 1.0$ \\
Young's modulus & $2500 \mathrm{MPa}$ \\
Density $\rho$ & $1.78 \times 10^{3} \mathrm{~kg} / \mathrm{m}^{3}$ \\
\hline
\end{tabular}

The flow boundary layers of the reverse trapezoidal cylinder and the square cylinder separate at the windward side. The air is directly acting on the windward side $D$ $(D>d)$. For these cases, the disturbance of trapezoidal and square cylinder to the flow field is obviously larger than that of triangular and trapezoidal cylinder. Especially when the flow speed $U \geq 4 \mathrm{~m} / \mathrm{s}$, the output voltage and power are rapidly increasing. When $U=6.3 \mathrm{~m} / \mathrm{s}$, the resonance phenomenon occurs, which leads the amplitude of the piezoelectric patch to increase suddenly. The energy conversion efficiency of reverse trapezoidal cylinder and square cylinder reaches the maximum values. Beyond critical wind speeds, the energy output of the reverse trapezoidal cylinder performed better for harvesting power. The maximum voltage and output power are $1.806 \mathrm{~V}$ and $16.30 \mu \mathrm{W}$, respectively. For the reverse trapezoidal cylinder and square cylinder, the separation points are fixed at the windward side and the separated shear layers have great influence on the pressure 


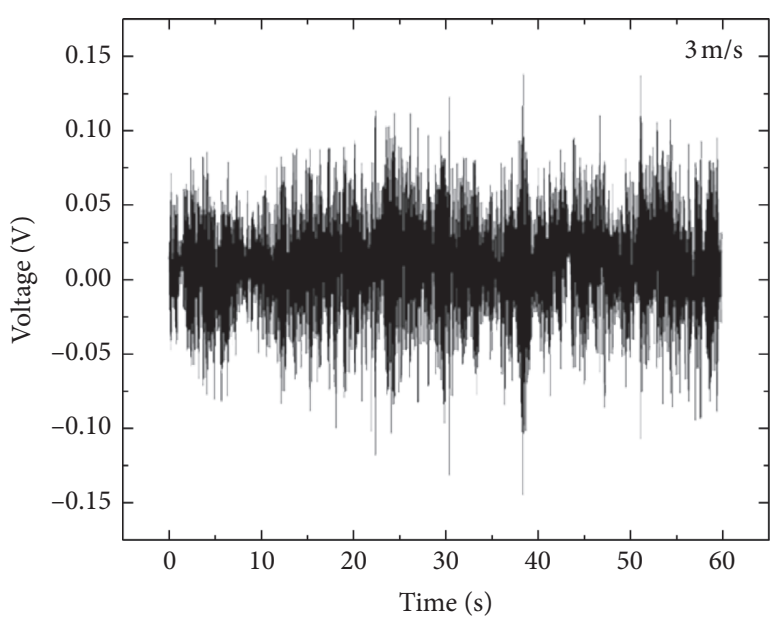

(a)

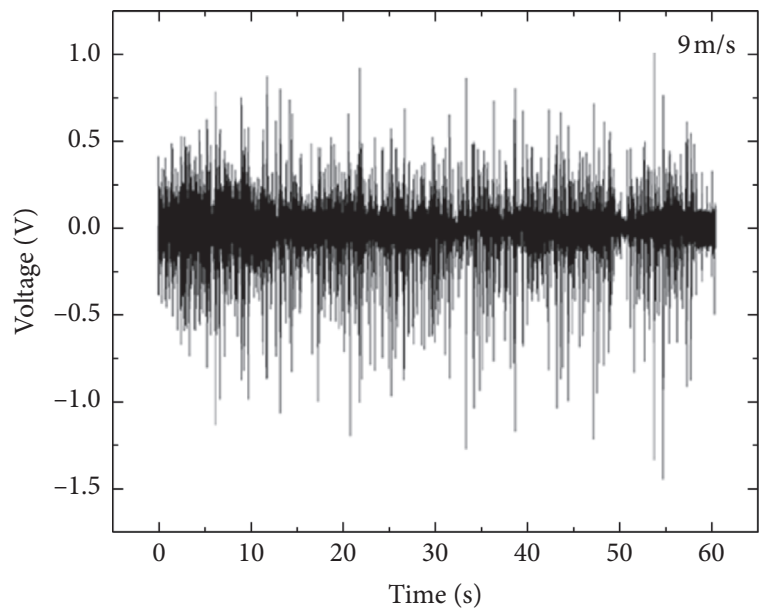

(c)

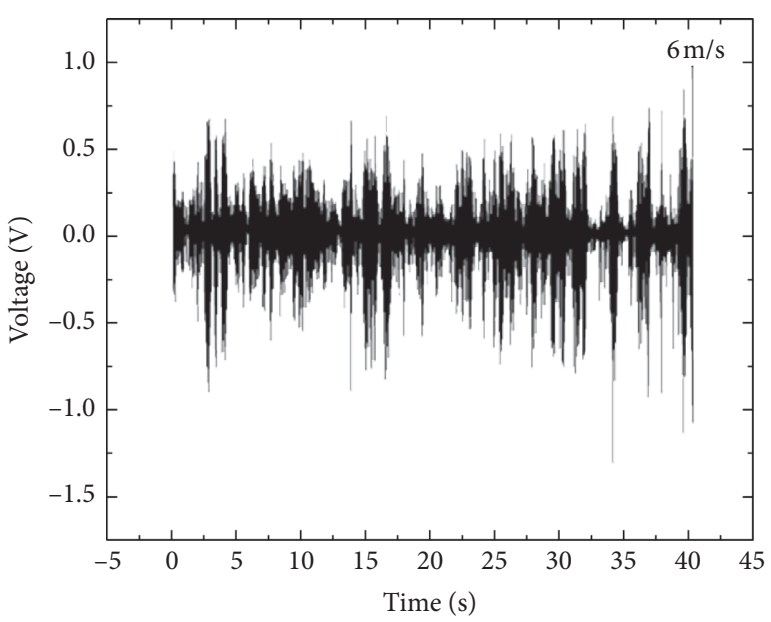

(b)

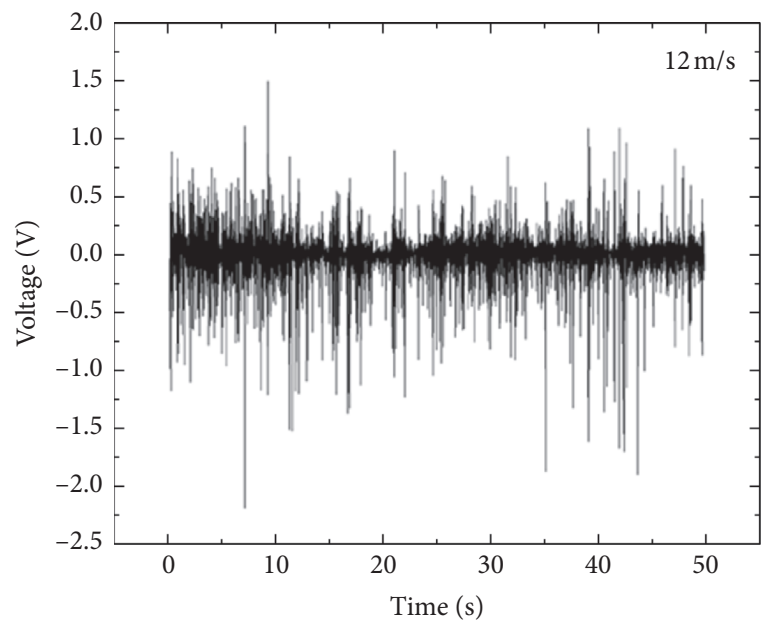

(d)

FIGURE 4: Voltage output of piezoelectric patch under different wind speed.

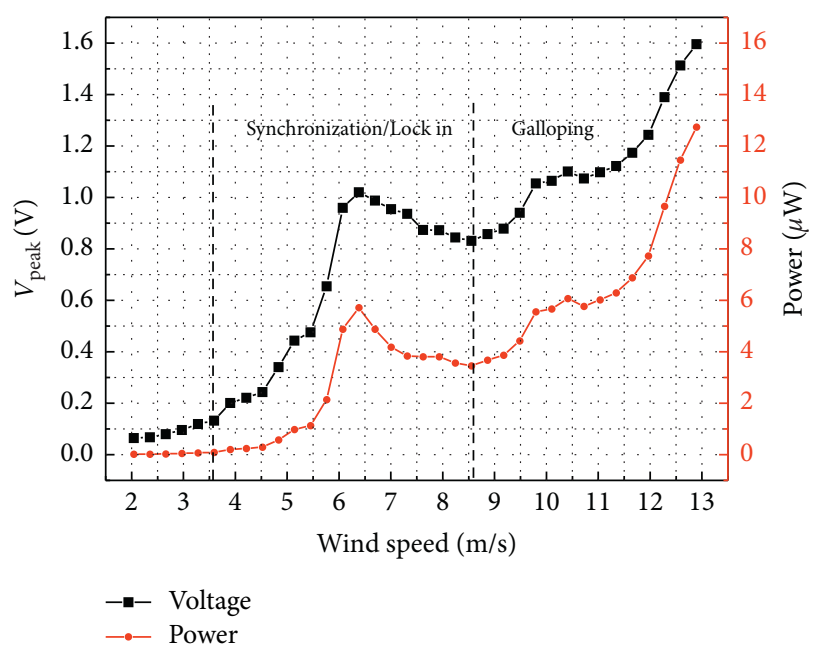

Figure 5: Mean peak voltage and power curve. 


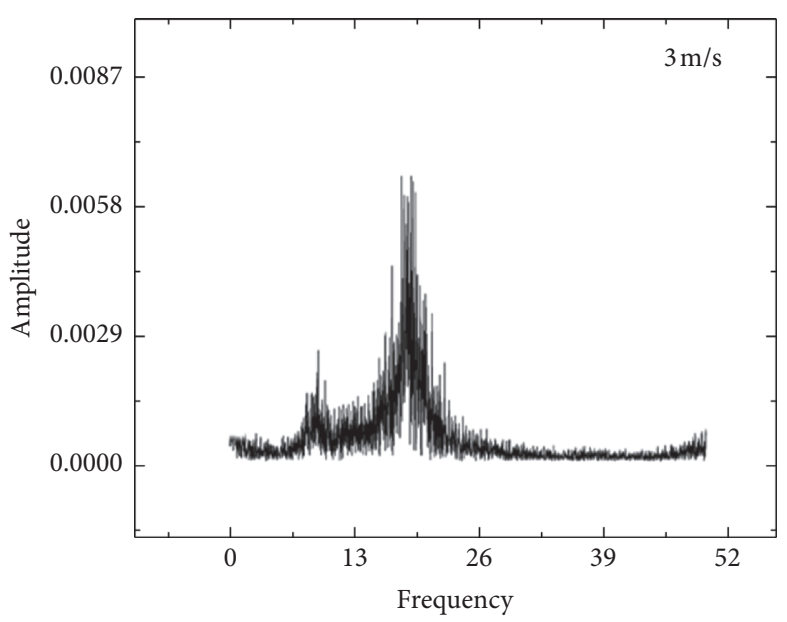

(a)

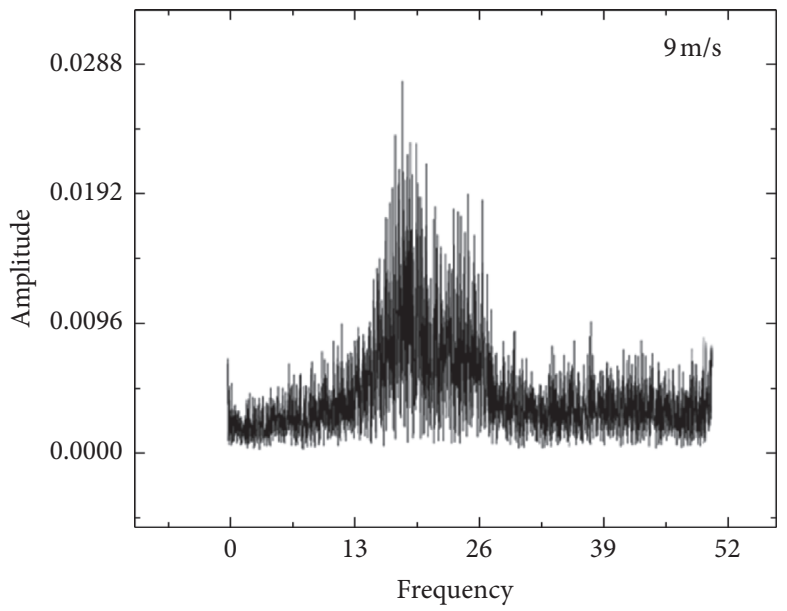

(c)

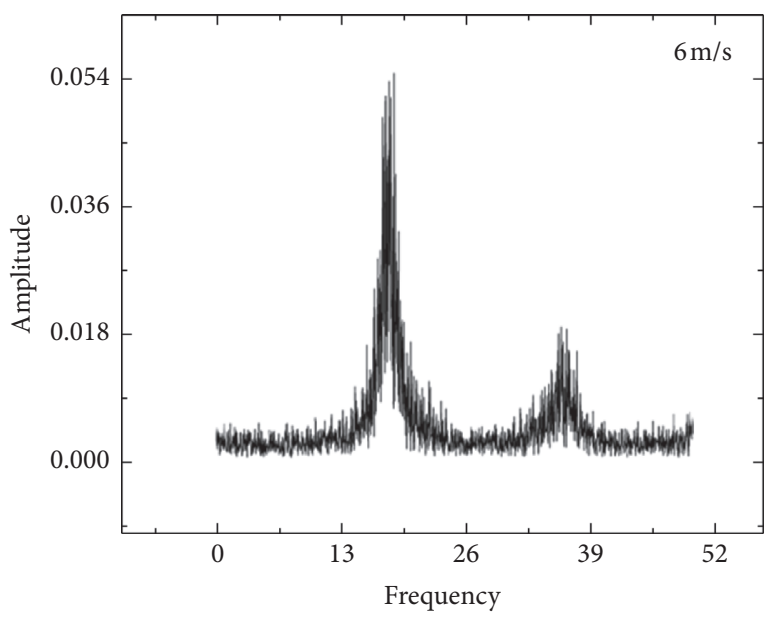

(b)

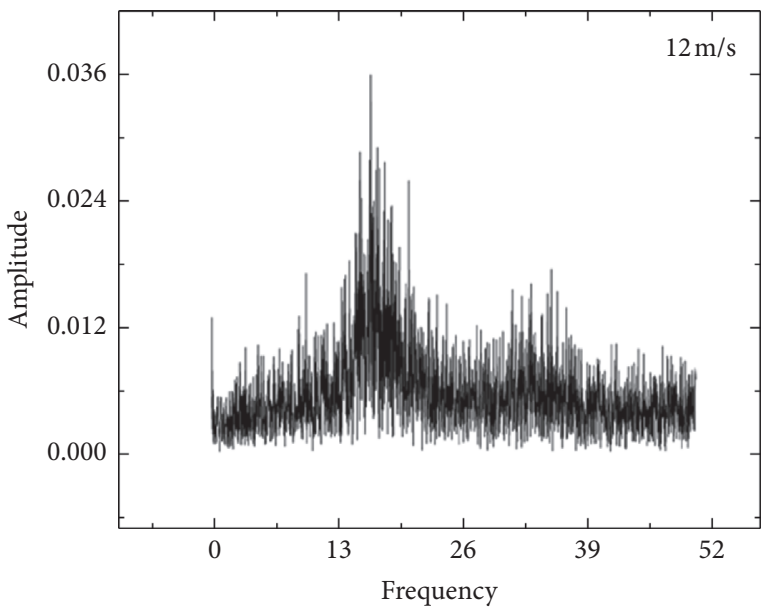

(d)

FIGURE 6: Voltage frequency spectra for piezoelectric patch under different wind speeds.

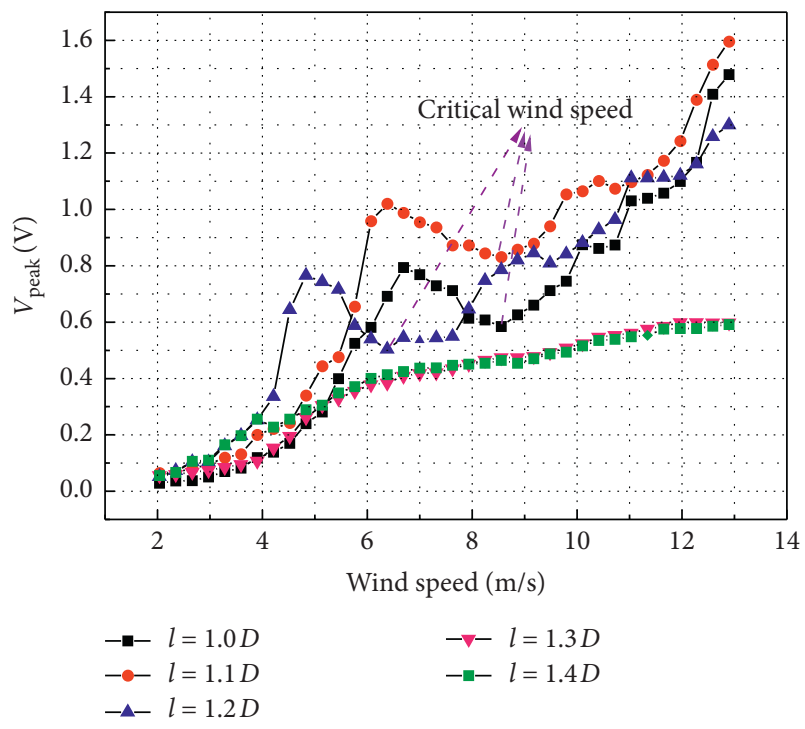

FIGURE 7: $V_{\text {peak }}$ of square cylinder with different piezoelectricpatch lengths.

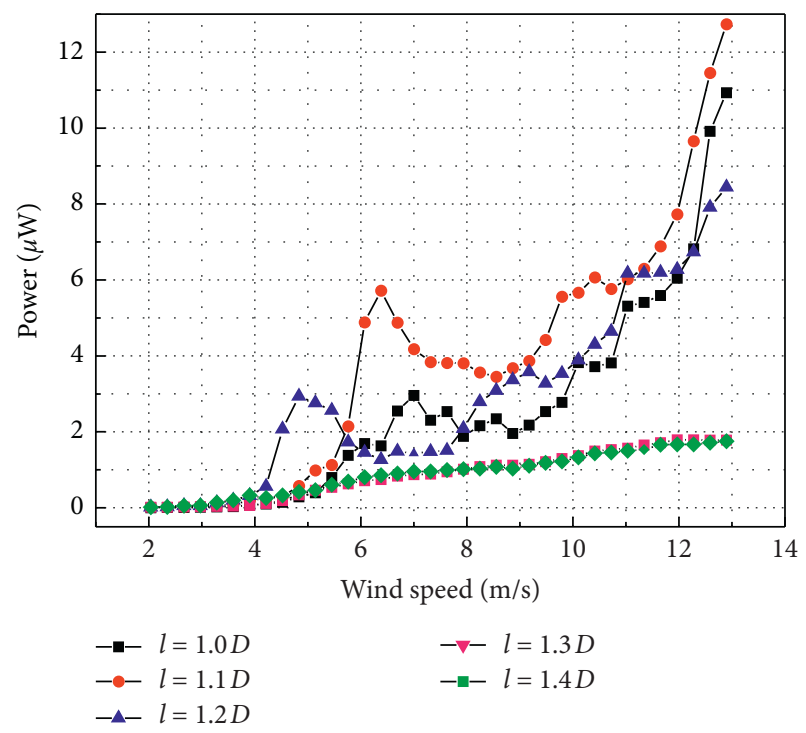

FIgure 8: Power of square cylinder with different piezoelectricpatch lengths. 


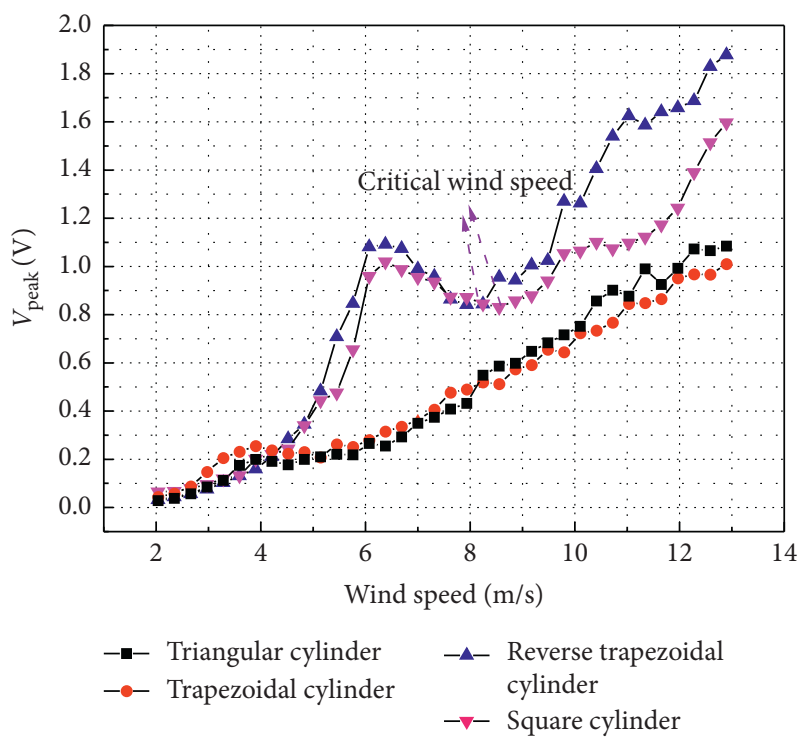

FIGURE 9: $\mathrm{V}_{\text {peak }}$ of the harvester with different cross-sectional geometries.

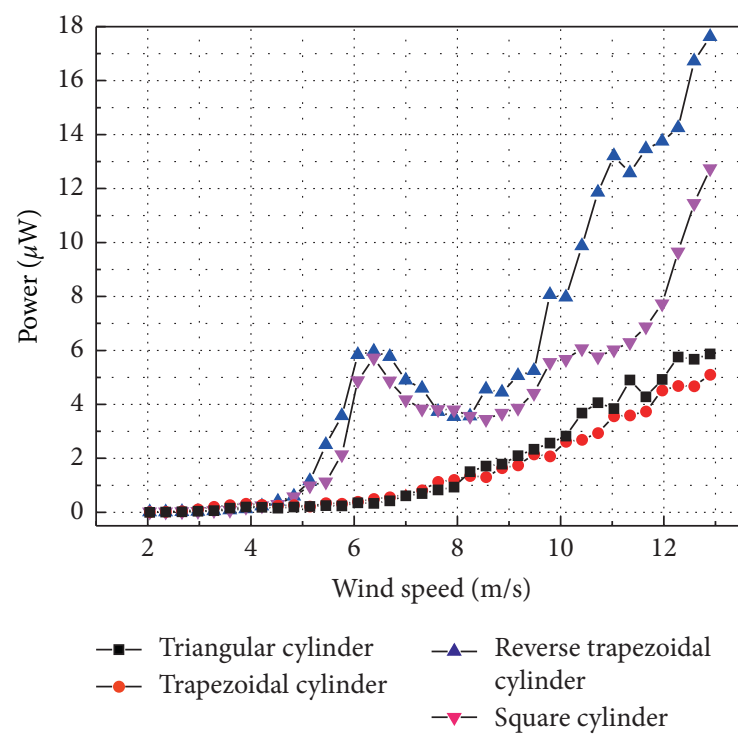

(a)

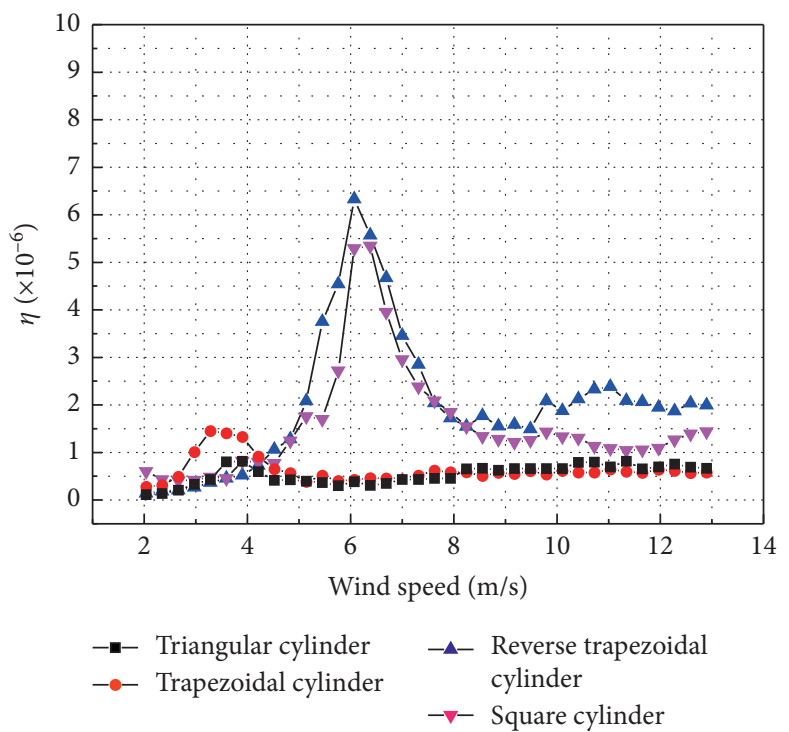

(b)

Figure 10: Power and conversion efficiency of the harvester with different cross-sectional geometries.

distribution around the after body, and then a large fluctuation in the lift force and a large vibration amplitude of the cylinder can be observed, so that the output voltage and energy of energy harvesters are higher.

Obviously, Figure 9 shows that the VIV and galloping phenomenon are observed for the reverse trapezoidal cylinder and the square cylinder. Figure 11 is plotted to see more clearly the working conditions of the VIV and galloping phenomenon. Apparently, this phenomenon is observed in the reverse trapezoidal and square cylinders when $l=1.0 D-1.2 D$. This phenomenon results in an improved efficiency of the harvester. Therefore, it is concluded that the occurrence of galloping is determined by the length of piezoelectric patch and the crosssectional geometry of bluff body. 


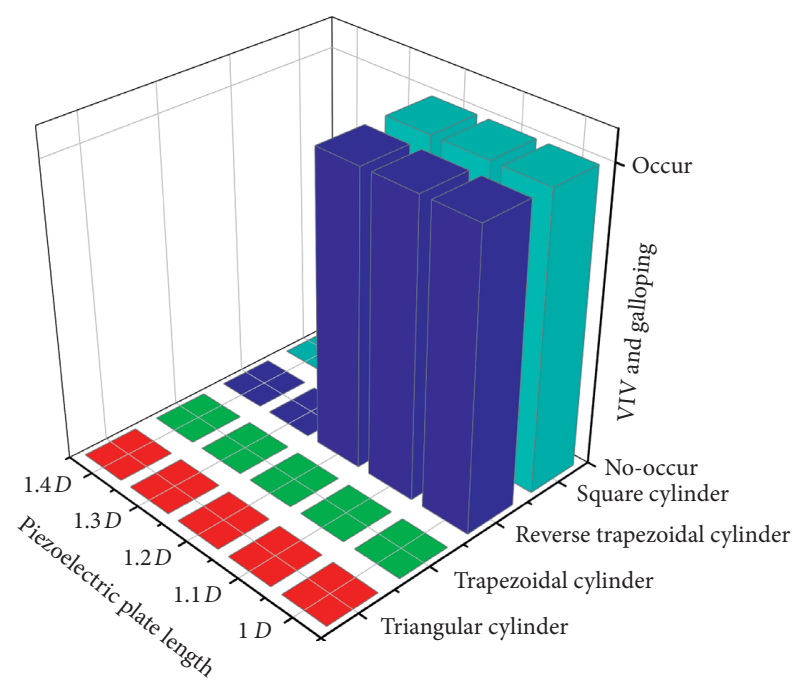

FIGURE 11: The phenomenon of VIV and galloping summary chart.

\section{Conclusions}

A series of experimental tests are performed in the wind tunnel to study the effects of body shape and piezoelectricpatch length on energy conversion by FIV in this work. The major conclusions are summarized as follows:

(1) Wind speed will significantly affect the voltage output of the harvester. For the reverse trapezoidal cylinder and square cylinder, the FIV can be divided into lock-in region and galloping region in the range of experimental wind speed. However, there is only one region for triangular cylinder and trapezoidal cylinder, where voltage monotonously increases as the wind speed increases. The reverse trapezoidal and square cylinders with $l=1.0 D-1.2 D$ can be continuously harvested when exceeding the critical wind speed.

(2) For the reverse trapezoidal cylinder and square cylinder, the VIV and galloping phenomenon can be observed at $l=1.0 D, l=1.1 D$, and $l=1.2 D$. For $l=1.0 D, l=1.1 D$, and $l=1.2 D$, the critical wind speed decreases as the length of the piezoelectric patch increases. In the range of experimental wind velocity, effect of the length of piezoelectric patch on the energy harvesting of reverse trapezoidal cylinder and square cylinder is small.

(3) Transition phase from VIV to galloping is featured by a small amplitude and high-frequency vibration. Galloping is featured by large amplitude and a lowfrequency vibration.

(4) Both of the length of the piezoelectric patch and the geometry of the bluff body are the key factors that affect the energy output. At $l=1.1 D$, the performance of energy harvester in reverse trapezoidal cylinder and square cylinder is better than that in triangular and trapezoidal cylinders. The maximum voltage and output power are $1.806 \mathrm{~V}$ and $16.30 \mu \mathrm{W}$, respectively.

\section{Data Availability}

The data used to support the findings of this study are included within the article.

\section{Conflicts of Interest}

The authors declare that there are no conflicts of interest regarding the publication of this paper.

\section{Acknowledgments}

This research was funded by the National Natural Science Foundation of China (Grant no. 51776021).

\section{References}

[1] S. Saadon and O. Sidek, "A review of vibration-based MEMS piezoelectric energy harvesters," Energy Conversion and Management, vol. 52, no. 1, pp. 500-504, 2011.

[2] M. R. Yuce, "Implementation of wireless body area networks for healthcare systems," Sensors and Actuators A: Physical, vol. 162, no. 1, pp. 116-129, 2010.

[3] X. Tang, X. Wang, R. Cattley, F. Gu, and A. Ball, "Energy harvesting technologies for achieving self-powered wireless sensor networks in machine condition monitoring: a review," Sensors, vol. 18, no. 12, p. 4113, 2018.

[4] F. K. Shaikh and S. Zeadally, "Energy harvesting in wireless sensor networks: a comprehensive review," Renewable and Sustainable Energy Reviews, vol. 55, pp. 1041-1054, 2016.

[5] M. T. Todaro, F. Guido, V. Mastronardi et al., "Piezoelectric MEMS vibrational energy harvesters: advances and outlook," Microelectronic Engineering, vol. 183-184, pp. 23-36, 2017.

[6] J. Bukala, K. Damaziak, K. Kroszczynski, M. Krzeszowiec, and J. Malachowski, "Investigation of parameters influencing the efficiency of small wind turbines," Journal of Wind Engineering and Industrial Aerodynamics, vol. 146, pp. 29-38, 2015.

[7] L. Ding, Q. Zou, L. Zhang, and H. Wang, "Research on flowinduced vibration and energy harvesting of three circular cylinders with roughness strips in tandem," Energies, vol. 11, no. 11, p. 2977, 2018.

[8] J. Wang, Z. Su, H. Li, L. Ding, H. Zhu, and O. Gaidai, "Imposing a wake effect to improve clean marine energy harvesting by flow-induced vibrationsflow-induced vibrations," Ocean Engineering, vol. 208, Article ID 107455, 2020.

[9] M. Zhou, M. Al-Furjan, and B. Wang, "Modeling and efficiency analysis of a piezoelectric energy harvester based on the flow induced vibration of a piezoelectric composite pipe," Sensors, vol. 18, no. 12, p. 4277, 2018.

[10] Z. L. Jin, G. P. Li, J. L. Wang, and Z. E. Zhang, "Design, modeling, and experiments of the vortex-induced vibration piezoelectric energy harvester with bionic attachments," Complexity, vol. 2019, Article ID 1670284, 13 pages, 2019.

[11] L. Ding, L. Yang, Z. Yang, L. Zhang, C. Wu, and B. Yan, "Performance improvement of aeroelastic energy harvesters with two symmetrical fin-shaped rods," Journal of Wind Engineering and Industrial Aerodynamics, vol. 196, Article ID 104051, 2020.

[12] J. Wang, L. Geng, L. Ding, H. Zhu, and D. Yurchenko, "The state-of-the-art review on energy harvesting from flow-induced vibrations," Applied Energy, vol. 267, Article ID 114902, 2020. 
[13] Z. Yang, L. Ding, L. Zhang, L. Yang, and H. He, “Two degrees of freedom flow-induced vibration and heat transfer of an isothermal cylinder," International Journal of Heat and Mass Transfer, vol. 154, Article ID 119766, 2020.

[14] T. K. Prasanth and S. Mittal, "Vortex-induced vibrations of a circular cylinder at low Reynolds numbers," Journal of Fluid Mechanics, vol. 594, pp. 463-491, 2007.

[15] M. Zhao, L. Cheng, H. An, and F. Tong, "Flow and flowinduced vibration of a square array of cylinders in steady currents," Fluid Dynamics Research, vol. 47, no. 4, Article ID 045505, 2015.

[16] Q. Zou, L. Ding, H. Wang, J. Wang, and L. Zhang, "Twodegree-of-freedom flow-induced vibration of a rotating circular cylinder," Ocean Engineering, vol. 191, Article ID 106505, 2019.

[17] H. Zhu, G. Li, and J. Wang, "Flow-induced vibration of a circular cylinder with splitter plates placed upstream and downstream individually and simultaneously," Applied Ocean Research, vol. 97, Article ID 102084, 2020.

[18] H. Zhu, W. Liu, and T. Zhou, "Direct numerical simulation of the wake adjustment and hydrodynamic characteristics of a circular cylinder symmetrically attached with fin-shaped strips," Ocean Engineering, vol. 195, Article ID 106756, 2020.

[19] M. M. Bernitsas, K. Raghavan, Y. Ben-Simon, and E. M. H. Garcia, "VIVACE (vortex induced vibration aquatic clean energy): a new concept in generation of clean and renewable energy from fluid flow," Journal of Offshore Mechanics and Arctic Engineering, vol. 130, no. 4, Article ID 041101, 2008.

[20] L. Ding, L. Zhang, E. S. Kim, and M. M. Bernitsas, "URANS vs. experiments of flow induced motions of multiple circular cylinders with passive turbulence control," Journal of Fluids and Structures, vol. 54, pp. 612-628, 2015.

[21] L. Ding, L. Zhang, C. Wu, E. S. Kim, and M. M. Bernitsas, "Numerical study on the effect of tandem spacing on flowinduced motions of two cylinders with passive turbulence control," Journal of Offshore Mechanics and Arctic Engineering, vol. 139, Article ID 021801, 2017.

[22] C. H. K. Williamson and R. Govardhan, "A brief review of recent results in vortex-induced vibrations," Journal of Wind Engineering and Industrial Aerodynamics, vol. 96, no. 6-7, pp. 713-735, 2008.

[23] G. W. Taylor, J. R. Burns, S. A. Kammann, W. B. Powers, and T. R. Welsh, "The energy harvesting eel: a small subsurface ocean/river power generator," IEEE Journal of Oceanic Engineering, vol. 26, no. 4, pp. 539-547, 2001.

[24] J. J. Allen and A. J. Smits, "Energy harvesting eel," Journal of Fluids and Structures, vol. 15, no. 3-4, pp. 629-640, 2001.

[25] F. Cellini, J. Pounds, S. D. Peterson, and M. Porfiri, "Underwater energy harvesting from a turbine hosting ionic polymer metal composites," Smart Materials and Structures, vol. 23, no. 8, Article ID 085023, 2014.

[26] R. Song, X. Shan, F. Lv, and T. Xie, "A study of vortex-induced energy harvesting from water using PZT piezoelectric cantilever with cylindrical extension," Ceramics International, vol. 41, pp. S768-S773, 2015.

[27] Y. Hu, B. Yang, X. Chen, X. Wang, and J. Liu, "Modeling and experimental study of a piezoelectric energy harvester from vortex shedding-induced vibration," Energy Conversion and Management, vol. 162, pp. 145-158, 2018.

[28] J. Wang, L. Tang, L. Zhao, and Z. Zhang, "Efficiency investigation on energy harvesting from airflows in HVAC system based on galloping of isosceles triangle sectioned bluff bodies," Energy, vol. 172, pp. 1066-1078, 2019.
[29] K. Yang, J. Wang, and D. Yurchenko, “A double-beam piezomagneto-elastic wind energy harvester for improving the galloping-based energy harvesting," Applied Physics Letters, vol. 115, no. 19, Article ID 193901, 2019.

[30] L. Wang and D. Zhu, "Enhancing output power of a cantilever-based flapping airflow energy harvester using external mechanical interventions," Sensors, vol. 19, no. 7, p. 1499, 2019.

[31] H.-X. Zou, L.-C. Zhao, Q.-H. Gao et al., "Mechanical modulations for enhancing energy harvesting: principles, methods and applications," Applied Energy, vol. 255, Article ID 113871, 2019.

[32] L.-C. Zhao, H.-X. Zou, G. Yan et al., "A water-proof magnetically coupled piezoelectric-electromagnetic hybrid wind energy harvester," Applied Energy, vol. 239, pp. 735-746, 2019.

[33] L.-C. Zhao, H.-X. Zou, G. Yan et al., "Magnetic coupling and flextensional amplification mechanisms for high-robustness ambient wind energy harvesting," Energy Conversion and Management, vol. 201, Article ID 112166, 2019.

[34] M. Zhang, G. Hu, and J. Wang, "Bluff body with built-in piezoelectric cantilever for flow-induced energy harvesting," International Journal of Energy Research, vol. 44, no. 5, pp. 3762-3777, 2020.

[35] G. Hu, K. T. Tse, M. Wei, R. Naseer, A. Abdelkefi, and K. C. S. Kwok, "Experimental investigation on the efficiency of circular cylinder-based wind energy harvester with different rod-shaped attachments," Applied Energy, vol. 226, pp. 682689, 2018.

[36] L. B. Zhang, H. L. Dai, A. Abdelkefi, and L. Wang, "Experimental investigation of aerodynamic energy harvester with different interference cylinder cross-sections," Energy, vol. 167, pp. 970-981, 2019.

[37] H. L. Dai, A. Abdelkefi, Y. Yang, and L. Wang, "Orientation of bluff body for designing efficient energy harvesters from vortex-induced vibrations," Applied Physics Letters, vol. 108, Article ID 053902, 195 pages, 2016.

[38] J. Wang, S. Zhou, Z. Zhang, and D. Yurchenko, "High-performance piezoelectric wind energy harvester with Y-shaped attachments," Energy Conversion and Management, vol. 181, pp. 645-652, 2019. 